\title{
User Context Aware Base Station Power Flow Model
}

\author{
Barbara Walsh ${ }^{\phi}$ and Ronan Farrell* \\ ${ }^{\dagger}$ RF and Mixed Signal Systems Research Group, \\ Department of Electronic Engineering, \\ NUI Maynooth, Maynooth, Co. Kildare \\ IRELAND \\ E-mail: ${ }^{\dagger}$ bwalsh@eeng.nuim.ie \\ * RF and Mixed Signal Systems Research Group, \\ Department of Electronic Engineering, \\ NUI Maynooth, Maynooth, Co. Kildare \\ IRELAND \\ E-mail:*rfarrell@eeng.nuim.ie
}

\begin{abstract}
At present the testing of power amplifiers within base station transmitters is limited to testing at component level as opposed to testing at the system level. While the detection of catastrophic failure is possible, that of performance degradation is not. This paper proposes a base station model with respect to transmitter output power with the aim of introducing system level monitoring of the power amplifier behaviour within the base station. Our model reflects the expected output levels of second or third generation CDMA base stations conforming to the Open Base Station Architecture Initiative (OBSAI) open base station reference architecture. The simulated base station output power is verified by comparison to field data using such metrics as power complementary cumulative distribution function (CCDF), volatility, absolute deviation, mean absolute deviation and rate of change.
\end{abstract}

\section{INTRODUCTION}

In recent years many base station manufacturers have begun to integrate other company's components into their base stations. These components often include some self test capabilities. Components, for example power amplifiers for base station transmitters, supplied by different $3^{\text {rd }}$ party vendors may have different self test capabilities and different reliability levels. Furthermore, it is difficult to use their disparate test functions within a system to provide standard and comprehensive tests across the full range of operational modes of the system. Because of this certain failure mechanisms and performance degradation may not be detected or false alarms may be reported. A desirable improvement on this state of affairs would be to develop a system level test. Power amplifiers are a particular case of a component that is prone to failure, and where partial failures are difficult to detect except through disruption of service. For a system level test, the expected output power level of the base station should be compared to a predicted output power level, the value of which would vary with the user context. As the first step in providing a context aware base station power alarm, a base station model focused on output power levels has been developed based on the Open Base Station Architecture Initiative (OBSAI) reference architecture for CDMA [1, 2]. Section II gives a brief overview of the OBSAI reference architecture. In section III the difficulties encountered in modelling the base station output power are discussed. The software implementation of the base station model is outlined in section IV. The metrics used to verify the results and the simulated results are presented in section VI. The conclusions are drawn in section $\mathrm{V}$. 


\section{OPEN BASE StATION MOdEL}

The OBSAI reference architecture consists of four functional blocks and three internal interfaces between the functional blocks as shown in Figure 1. Each block represents a logical separation of the base station functions and each block consists of one or more modules, which perform a subset of block functions. The open base station reference consists of the following functional blocks:

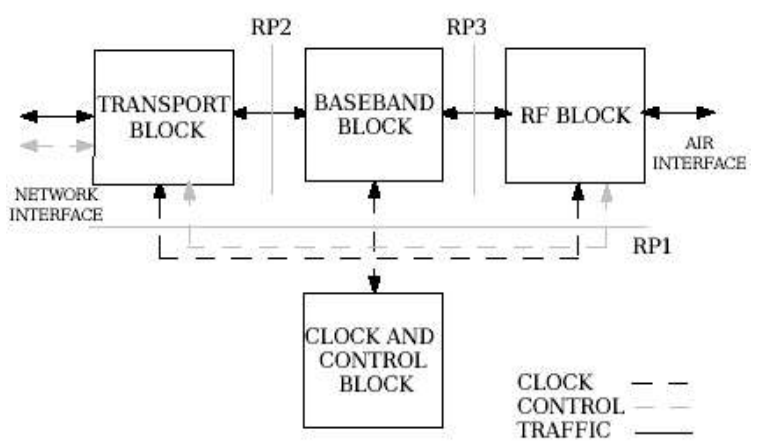

Figure 1: Base Station Reference Architecture

\section{a) Transport Block}

The transport block performs interfacing functions between the rest of the base station and the base station controller. It enables the communication of data from external networks such as the public telephone network or the internet to the base station. It facilitates the transmission of control data between the clock and control block and the base station controller. User data is also interchanged between external networks and the baseband block via the transport block. Aditionally administrative functions such as quality of service and security are carried out by the transport block [1].

\section{b) Control and Clock Block}

The clock and control block controls all base station activities, resources and monitors its performance. Its main function is radio resource management. This function handles the setting up of radio links, the admission or denial of new users on the basis of available resources and the allocation of system resources. The generation and distribution of the clock signal is another important function of the clock and control block [1].

\section{c) Baseband Block}

The baseband block performs various digital signal processing functions on the user data stream sent to it by the transport block. For CMDA base stations the data stream is encoded, interleaved, assigned a long code which identifies the intended receiver, a walsh code which identifies the transmission channel and a pseudonoise code which identifies the cell in which the base station is located. These functions prepare the the signal for transmisson so that it can be reliability detected by the receiver. The digital data stream is then sent to the RF block for transmission. [1].

\section{d) $R F$ Block}

The RF block performs some final signal processing on the signal received from the transport block before transmission occurrs. Digital to anaolg conversion, upconversion, power amplification. power combining and transmit filtering are carried out on the digital signal received from the baseband block. The signal is then transmitted by the RF block [1].

\section{e) Internal Interfaces}

The reference points (RP) are interfaces for the transport of signaling and user data between modules. RP1 is the internal interface between the clock and control block and the other blocks and interchanges control data and clock signals. RP2 is the interface for the transport of user data between the transport block and the baseband block. Neither of these interfaces have an effect on the output power levels of the RF module. RP3 transports the user data between the baseband block and the RF block. RP3 has a significant effect on the output of the RF block. Several topologies for the interface between the baseband and RF blocks are allowed by the OBSAI reference architecture. They specify the connectivity between the baseband and RF blocks and include mesh as shown in Figure 2, centralized combiner and distributor shown in Figure 3 as well as bridge modules connecting several modules of the same type together.

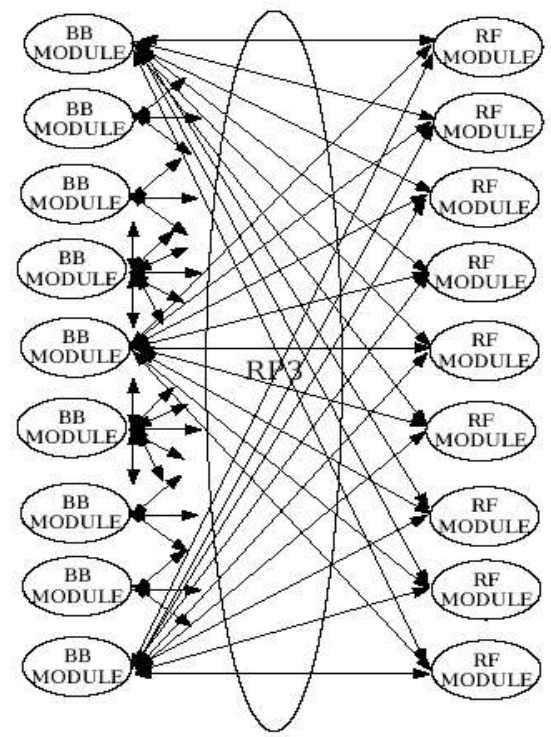

Figure 2: Full Mesh Interface Connecting K Baseband Modules to K RF Modules. All Links Are Active And Transport Data. 
Each of these topologies have an effect on the final output power as the number of connections between RF and baseband blocks differs depending on the interface topology. This will be discussed further in section III $[1,2]$.

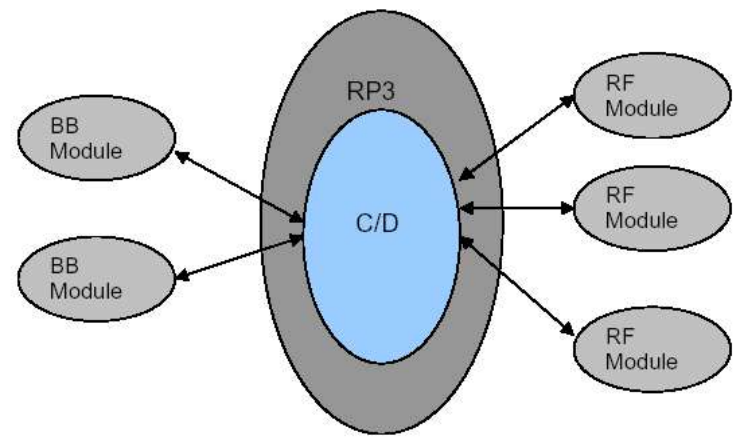

Figure 3: Centralised Combiner and Distributor RF Interface.

\section{MODELLING ISSUES}

There are many difficulties involved in modelling a base station with respect to power. These difficulties arise due to the fact that the user load is not constant. It varies with:

- The number of users.

- The mobility of the users.

- The type of traffic (either voice or data).

- The data rate of signal transmision.

- The RF interface topology being used.

- The distance of the user from the base station.

\section{a) User Statistics}

To model typical call statistics certain assumptions were made regarding transmitter usage. It is assumed that the average number of calls and text messages per minute depends on the time of day or night and on whether it is mid week or the weekend. It is also surmised that the majority of the traffic is voice as opposed to data. Several user contexts were modelled to reflect the varying user load. The first context models office hours with a large number of calls of short duration and a large proportion of nonstationary users. The second context replicates offpeak traffic with fewer calls placed on average but with calls of longer duration and a large proportion of stationary users. The final context represents the user load at quiet times where there are very few calls placed[3].

\section{b) Markov Chains}

Because of the limited bandwidth available in wireless cellular systems voice signals must be transmitted at low data rates. Voice signals have a lot of inherent redundency and so can be compressed by a vocoder to reduce the data rate. The output data rate of the vocoder varies on a frame by frame basis and is dependent on the user's voice activity. The output power at which the signal is transmitted is dependent on the output data rate of the vocoder [4, 5]. This output power variation with data rate is modelled using Markov transition matrices [3, 6, 7]. For a vocoder with four possible output data rates the transition matrix would be given by:

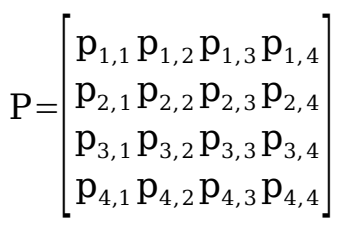

The elements of the matrix $\mathrm{P}$ are called transition probabilities. The transition probability $\mathrm{P}_{\mathrm{i}, \mathrm{j}}$ is the probability of transition from rate $i$ to rate $j$ where the maximum rate is one.

\section{c) RF Interface}

Each RF and baseband module can have a maximum of $\mathrm{K}$ pairs of unidirectional links connecting them to one another. A pair constitutes one incoming and one out going signal. Each RF module can implement $\mathrm{K}_{\mathrm{RFIN}}$ incoming links and $\mathrm{K}_{\text {RFOUT }}$ out going links with unused links disabled for power conservation purposes where

$$
\begin{aligned}
& 0 \leqslant \mathrm{~K}_{\text {RFIN }} \leqslant \mathrm{K}_{\text {MAX }} \\
& 0 \leqslant \mathrm{~K}_{\text {RFOUT }} \leqslant \mathrm{K}_{\text {MAX }} \\
& \mathrm{K}_{\text {MAX }} \leqslant 9
\end{aligned}
$$

with the maximum value for $\mathrm{K}$ set by the OBSAI reference architecture standard. Similarly the base band module can have a maximum of $\mathrm{K}$ pairs or unidirectional links with unused links disabled for power conservation.

$$
\begin{aligned}
& 0 \leqslant \mathrm{~K}_{\text {BBIN }} \leqslant \mathrm{K}_{\text {MAX }} \\
& 0 \leqslant \mathrm{~K}_{\text {BBOUT }} \leqslant \mathrm{K}_{\text {MAX }} \\
& \mathrm{K}_{\text {MAX }} \leqslant 9
\end{aligned}
$$

Assuming $\mathrm{N}$ baseband and M RF modules in a base station there are $\mathrm{N}^{*} \mathrm{M}$ pairs of unidirectional links in total between baseband and RF modules in full mesh as seen in Figure 2. Each baseband module is connected to M RF modules while every RF module is connected tp $\mathrm{N}$ basband modules. Assuming $\mathrm{N}$ baseband and M RF modules in a base station, all links from both baseband and RF modules are connected to a centralized combiner and distributor that is located in RP3. Inputs that are targeted for the same antenna and carrier at the same instant are added together so that a single output stream is formed. At a maximum $\mathrm{K}^{*}(\mathrm{~N}+\mathrm{M})$ pairs of unidirectional links are connected to the combiner 
and distributor as seen in Figure 3 but any number of links below this maximum can be connected [2]. Therefore the input power to a RF module, $\mathrm{P}_{\mathrm{RFIN}}$, for either topology is:

$$
\mathrm{P}_{\text {RFIN }}=\sum_{\mathrm{i}=1}^{\mathrm{K}} \mathrm{K}_{\text {RFIN }}(\mathrm{i}) * \mathrm{P}_{\text {CONNECTION }}(\mathrm{i})
$$

where $\mathrm{P}_{\text {CONNECTION }}$ is the power per uniderectional link. If there is more than one RF module then the total input power, $\mathrm{P}_{\text {RFINTOTAL}}$, between all the modules is:

$$
\mathrm{P}_{\text {RFINTOTAL }}=\sum_{\mathrm{i}=1}^{\mathrm{M}} \mathrm{P}_{\text {RFIN }}(\mathrm{i})
$$

\section{d) Power Variation With User Distance}

When considering the power variation with distance two cases should be taken into consideration: that of a mobile unit near the base station and that of a mobile unit at the boundary of the cell. For a mobile unit close to the base station the transmitted power, $\mathrm{P}_{\mathrm{j}}$, at the base station for the jth mobile unit is modelled using the model for signal propagation in free space. It is given by:

$$
P_{j} \propto r_{j}^{n}
$$

where $r_{j}$ is the distance between the cell site and the $\mathrm{jth}$ mobile unit and $\mathrm{n}$ is a number. It was found that the power control scheme with $n=2$ [8] provides the optimum capacity and also meets the requirements that the forward link signal still reach the mobile unit near the boundary of the cell with reduced power to reduce interference with adjacent cells. This provides a model for the variation of output power with distance from the base station as follows:

$$
P_{j}=P_{R}\left(\frac{r_{j}}{R}\right)^{2}
$$

where $P_{R}$ is the power required to reach those mobile units at the boundary at the cell boundary.

\section{e) Modelling Speech Input Signal}

The speech input signal is modelled as a signal which varies in amplitude for the duration of the call. The variation of the speech signal follows a poisson distribution. A typical input signal for one user is shown in Figure 4 above.

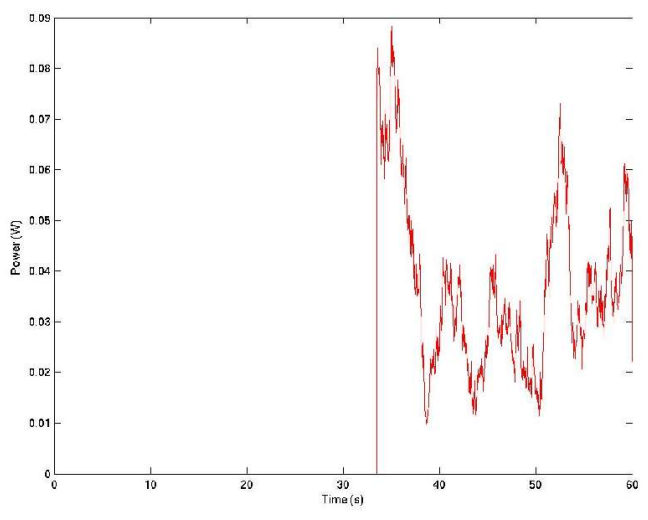

Figure 4: Input Speech Signal

\section{SOFTWARE IMPLMENTATION}

The base station was modelled using MATLAB. Initially the model was designed to reflect a IS-95 based system and was then extended to cover CDMA2000. First, the system parameters such as user type, context, distance of user from the base station, number of users, call duration etc. are initialised. Values for these parameters were selected at random from a certain interval which reflected the user context being simulated. In the case of certain variables a poisson distribution was assumed. The output power for each call was then calculated depending on whether the user was stationary or non-stationary. These calculations take into consideration distance, data rate, number of power amplifiers in the transmitter, interface topology and the mobility of the user. The combined output power is given by:

$$
\mathrm{P}_{\text {OUT }_{\text {тотаL }}}=\sum_{\mathrm{i}=1}^{\mathrm{n}} \mathrm{P}_{\text {OUT }}(\mathrm{i})
$$

Where $\mathrm{P}_{\text {оutтotal }}$ is the total output of the base station and $\mathrm{P}_{\mathrm{OUT}}(\mathrm{i})$ is the output of user $\mathrm{i}$. For non-stationary users the possibility of handover and the changing distance from the base station must also be considered. The changing distance of the user from the cell site is calculated by:

$$
\mathrm{d}_{\mathrm{t}+1}=\mathrm{d}_{\mathrm{t}} \pm\left(\mathrm{s}_{\mathrm{USER}} * \Delta \mathrm{t}\right)
$$

Where $\mathrm{d}$ is distance from the base station and SUSER is the speed of the user. For users moving away from the base station the duration to handover is given by:

$$
\mathrm{t}_{\text {HANDOVER }}=\frac{\mathrm{d}_{\text {MAX }}-\mathrm{d}_{\text {INITIAL }}}{\mathrm{s}_{\text {USER }}}
$$

For users moving towards the base station it is given by:

$$
\mathrm{t}_{\text {HANDOVER }}=\frac{\left(2 * \mathrm{~d}_{\text {INITIAL }}\right)+\left(\mathrm{d}_{\text {MAX }}-\mathrm{d}_{\text {INITIAL }}\right)}{\mathrm{s}_{\text {USER }}}
$$


Where $\mathrm{d}_{\mathrm{MAX}}$ is the maximum distance from base station before handover occurs, $d_{\text {INITIAL }}$ is the initial distance of the user from the base station, SUSER is speed of user and $t_{\text {HANDOVER }}$ the time that will ellapse before handover occurs. System overhead in the form of control channels such as the pilot, paging and synchronization channel was also simulated. The output power of these channels does not vary much [3] and can be modelled as follows:

$$
\begin{aligned}
& \mathrm{P}_{\text {OVERHEAD }}=\mathrm{P}_{\text {PILOT }}+\mathrm{P}_{\text {SYNCH }}+\mathrm{P}_{\text {PAGE }} \\
& \mathrm{P}_{\text {OVERHEAD }} \approx 0.22 * \mathrm{P}_{\text {OUT }_{\text {TOTAL }}}
\end{aligned}
$$

The transport and clock and control blocks are not considered in this model as they do not affect the output power of the RF module. The overall simulation procedure is shown in the flowchart in Figure 5 below.

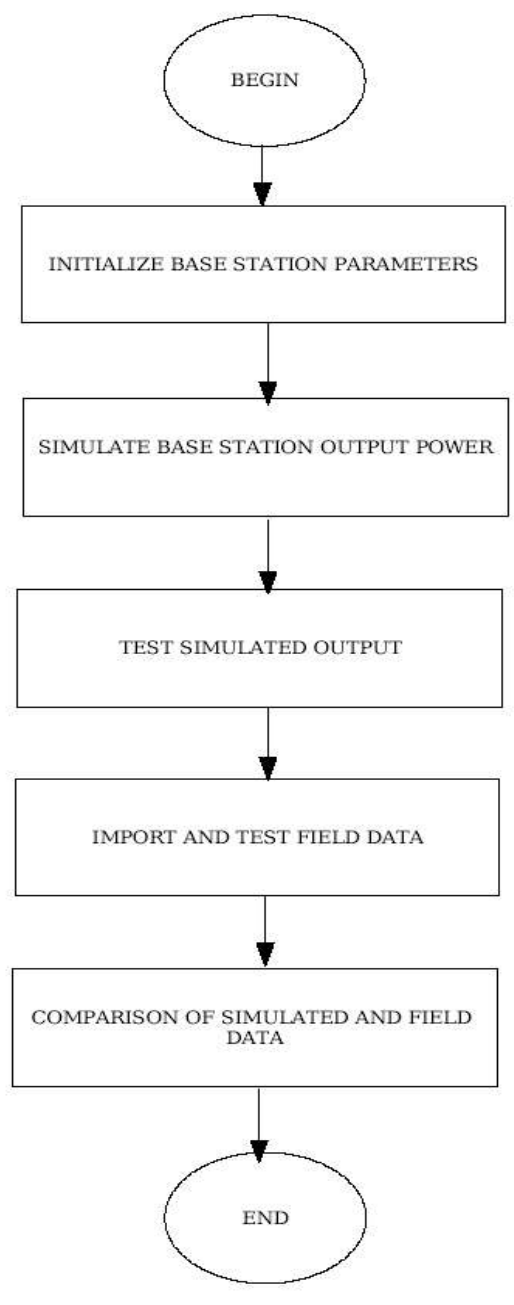

Figure 5: Model flowchart

\section{RESULTS}

The metrics chosen to run a comparsion between the simulated data and the field were the complementary cumulative distribution function, volatility, absolute deviation and rate of change. These metrics were chosen to evaluate the simulated output power profile as they indicate whether or not it has the same behavioural characteristics as the field data.

\section{a) Rate of Change}

The rate of change of output power with respect to time is calculated as follows:

$$
\Delta \mathrm{P}_{\text {OUT }}=\frac{\mathrm{P}_{2}-\mathrm{P}_{1}}{\Delta \mathrm{t}}
$$

It is then averaged over the simulation length. The value selected for $\Delta \mathrm{t}$ is $20 \times 10^{-3}$ seconds [4], the basic frame length for IS-95 and CDMA2000.

\section{b) Power Complementary Cumulative Distribution Function $(P C C D F)$}

An RF output power signal such as that shown in Figure 6 is difficult to quantify because of its inherent randomness. In order to extract useful information from the noise like signal a statistical description of the power level is needed. The PCCDF curves specify completely and without ambiguity the power characteristics of signals output by communications systems. The position of the PCCDF curve indicates the degree of peak-toaverage deviation of the signal in decibels. Signals with greater peak-to-average deviation are further to the right. The PCCDF was calculated by obtaining the PDF of the RF envelope and then computing its CDF. The PCCDF is the complement of the CDF [9].

$$
\mathrm{PCCDF}=1-\int \mathrm{PDF}(\mathrm{RF} \text { Envelope })
$$

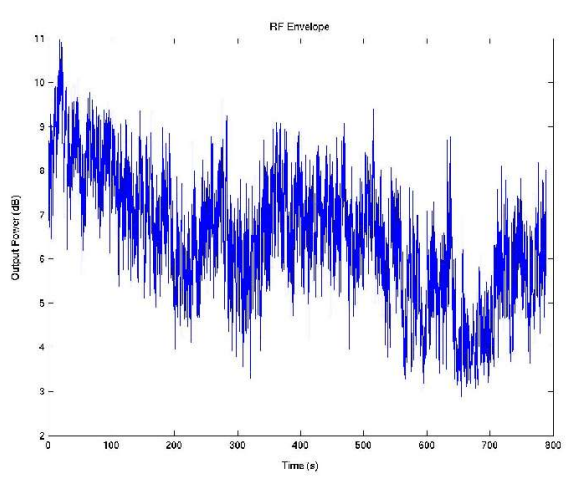

Figure 6: RF Envelope 


\section{c) Volatility}

Volatility is a measure of the random variations of the RF envelope stated as a percentage. Let

$$
\ldots \mathrm{Q}_{\mathrm{t}-2}, \mathrm{Q}_{\mathrm{t}-1}, \mathrm{Q}_{\mathrm{t}}, \mathrm{Q}_{\mathrm{t}+1}, \ldots
$$

be a stochastic process. The volatility [10] of the process at time $t$ is the standard deviation of $R_{t}$ and is calculated as follows:

$$
\begin{aligned}
& \mathrm{R}_{\mathrm{t}}=\ln \frac{\mathrm{Q}_{\mathrm{t}}}{\mathrm{Q}_{\mathrm{t}-1}} \\
& \mathrm{R}_{\mathrm{m}}=\frac{1}{\mathrm{n}} \sum_{\mathrm{i}=1}^{\mathrm{n}} \mathrm{R}_{\mathrm{t}} \\
& \mathrm{V}=\sqrt{\frac{\sum_{\mathrm{i}=1}^{\mathrm{n}}\left(\mathrm{R}_{\mathrm{t}}-\mathrm{R}_{\mathrm{m}}\right)^{2}}{\mathrm{n}-1}}
\end{aligned}
$$

where $R_{t}$ is the change in the output power at intervals of $20 \times 10^{-3}$ seconds. $R_{m}$ is the average change in output power over a period of 60 seconds. The volatility, $\mathrm{V}$, is calculated over a 60 second period and indicates how far output power deviates from the average $R_{m}$. A moving average for the volatility of the output power was then calculated for the duration of the simulation.

\section{d) Absolute Deviation}

The absoulute deviation provides a measure of the width of the distribution of the output power levels. Let $u$ denote the mean of a set of quantities $u_{i}$. The absolute deviation is defined by:

$$
\Delta \mathrm{u}_{\mathrm{i}}=\left|\mathrm{u}_{\mathrm{i}}-\overline{\mathrm{u}}\right|
$$

The mean absolute deviation of a set $\left\{\mathrm{u}_{0}, \mathrm{u}_{1}, \ldots, \mathrm{u}_{\mathrm{n}-1}\right\}$ is:

$$
\operatorname{mean} \Delta \mathrm{u}_{\mathrm{i}}=\frac{\sum_{\mathrm{i}=1}^{\mathrm{n}-1}\left|\mathrm{u}_{\mathrm{i}}-\overline{\mathrm{u}}\right|}{\mathrm{n}}
$$

The absolute deviation was calculated at $20 \times 10^{-3}$ second intervals. As the absolute deviation is constantly varying, a 60 second running average for the absolute deviation was computed over the simulation length.

\section{DISCUSSION}

A comparison between the simulated and measured data for each of these metrics can be seen in Figures 7 - 10 below. The analysis is based on the comparsion of 10 samples of data measured over a period of 4.5 minutes with 4.5 minutes of simulated data. As can be seen in Figure 7 the peak-to-average deviation of the simulated data is very close to that of the measured data. Figure 8 shows the percentage volatility in the form of a running average over the simulated length for both the real and simulated data. It is evident that variation of both the simulated data and measured data lie within the same interval. The level of absolute deviation from the mean value is displayed in Figure 9 in the form of a running average. Once again the levels of variation between the real and simulated are very close. The average rate of change over the simulation length is shown in Figure 10, with the simulated data reflecting comparable values to the measured data.

\section{CONCLUSIONS}

In this paper a base station model with respect to output power is presented. This model is compared to field data in terms of PCCDF, volatility, absolute deviation and rate of change. It has been shown that the model reflects similar PCCDF functions as the measured data. The rates of change, absolute deviation and volitility also lie within the same range as that of the field data. It can be concluded that the base station model presented in this paper accurately models the performance with respect to power of a real system. Using this model the system level response to the failure or performance degradation of a power amplifier in the base station transmitter with variable user load can be predicted. This will enable the development of dynamic amplifier performance monitoring at the system level within the basestation.

\section{ACKNOWLEDGEMENT}

This work has been supported through the Centre for Telecommunications Value-Chain Research (CTVR) and Science Foundation Ireland.

\section{REFERENCES}

[1] OBSAI. "Open Base Station Architecture Initiative BTS System Reference Document Version 1.0". OBSAI, 2002.

[2] OBSAI. "Reference Point 3 Specification Version 1.0". OBSAI, 2004.

[3] Private correspondance between Ronan Farrell and Greg Nardozza (Bell Labs), 2005.

[4] K. Wesołowski. "Mobile Communication Systems”. John Wiley \& Sons, 2002.

[5] Mischa Schwartz. "Mobile Wireless Communications". Cambridge University Press, 2005.

[6] O. Häggström. "Finite Markov Chains and Algorithmic Applications". Cambridge University Press, 2002. 
[7] R. De Guadenzi. “ Payload Nonlinearity Impact on the Globalstar Forward Link Multiplex Part II: System Analysis". IEEE Transactions on Vehicular technology 48:977-984, 1999.

[8] W.C.Y. Lee. "Overview of Cellular CDMA". IEEE Transactions on Vehicular Technology, 40:291-302, 1991.

[9] "Characterizing Digitally Modulated Signals with CCDF Curves". Agilent Application Note, 2000.

[10] K. R. Jain. "Putting Volatility to Work". Active Trader, April 2001.

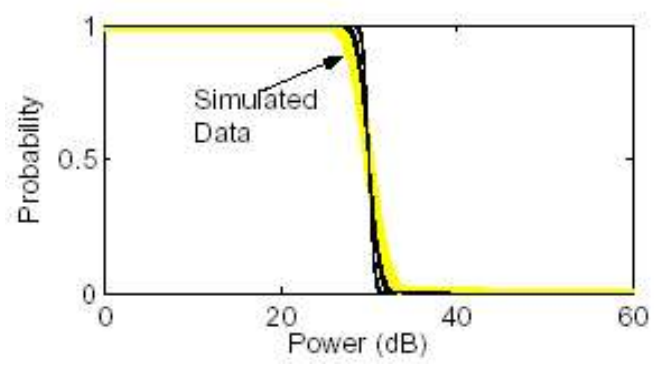

Figure 7: CCDF Curves

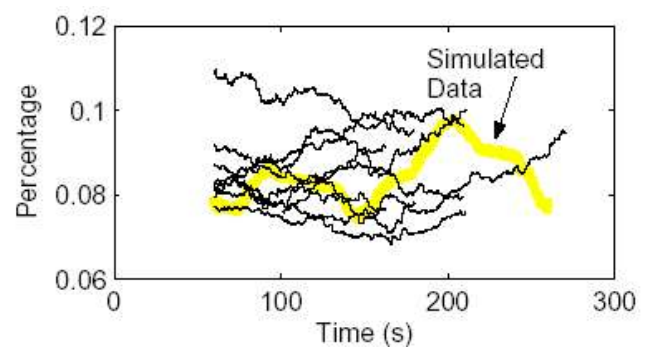

Figure 8: Volatility Curves

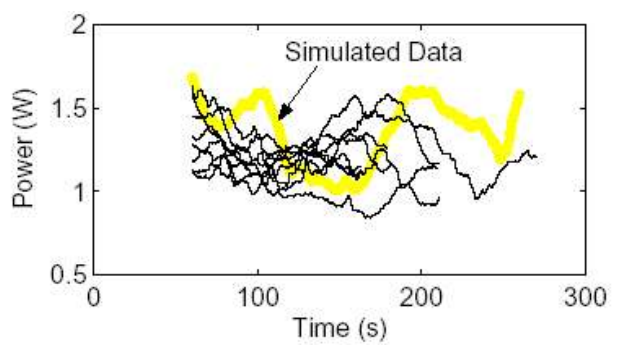

Figure 9: Absolute Deviation Curves

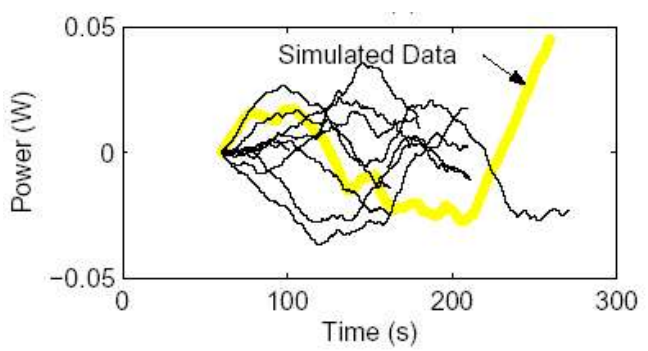

Figure 10: Rate of Change Curves 